and bile acid-lowering drugs, their current clinical practice was not always safety. Especially when using two or more hepatoprotective drugs, should pay more attention to whether it will aggravate the risk of adverse reactions or even allergic reactions. Results Based on the literature reports and the corresponding drug instructions, it is concluded that all three drugs might cause allergic reactions, but there are few reports of allergic reactions caused by ursodeoxycholic acid tablets. So far, there have been no reports of two allergic reactions for three drugs in the same patient in a short period. In this case, we judged that the first allergic reaction was caused by reduced glutathione for injection, and the second allergic reaction was caused by inosine injection, but the combination of ursodeoxycholic acid tablets may aggravate the allergic reaction.

Conclusions 1. Reduced glutathione for injection may lead to severe allergic reactions in women in the third trimester of pregnancy. 2. Ursodeoxycholic acid tablets may aggravate the risk of allergic reactions for inosine injection when combined with both drugs is used to protect liver and reduce bile acid levels in women in the third trimester of pregnancy.

\section{IDDF2019-ABS-0037 REBLEEDING AND PROGNOSIS IN CIRRHOTIC PATIENTS AFTER ERADICATION OF ESOPHAGOGASTRIC VARICES BY ENDOSCOPIC SEQUENTIAL THERAPY: A TERTIARY CENTRE COHORT STUDY}

Xing Wang ${ }^{*}$, Feng-Ping Zheng, Zhuo-Fu Wen, Hong Tian, Xiu-Qing Wei, Yun-Wei Guo, Jian-Zhong Li, Xiao-Liang Chen, Jin Tao, Jin-Ni Luo, Xiao-Ying Wu, Bin Wu. Department of Gastroenterology, The Third Affiliated Hospital of Sun Yat-sen University, Guangzhou, China

\subsection{6/gutjnl-2019-IDDFabstracts.246}

Background Esophagogastric variceal bleeding (EGVB) is a severe complication of liver cirrhosis and variceal eradication by modern endoscopic strategies may improve the recurrence and prognosis. Scant data is available about long-term observation after EGV eradication. We reported the incidence of rebleeding, variceal recurrence and mortality by endoscopic sequential therapy (EST), and investigated risk factors associated with prognosis.

Methods We carried out a retrospective cohort study recruiting consecutive cirrhotic patients with EGV who were regularly followed up in our endoscopy centre from Oct 2011 to June 2018. EST was defined as a combination strategy of band ligation, histoacryl injection and sclerotherapy. All patients had undergone EST until complete variceal eradication. Relevant medical and endoscopic data were collected. Rebleeding, variceal recurrence and all-cause mortality were considered as major outcomes. We calculated cumulative incidences of the outcomes and used Cox proportional hazard model to estimate hazard ratio (HR) and to identify risk factors.

Results We identified 468 patients for screening and 291 of them were finally included for analysis. The mean follows up duration was 32.4 months. After eradication of EGV, rebleeding was identified in 29 patients, and the cumulative incidence of rebleeding at 1 year, 2 year and 5 year was 3.4\%, 6.5\% and $9.6 \%$ respectively. Variceal recurrence was observed in 49 patients while the cumulative incidence at 1 year, 2 year and 5 year was $5.5 \%, 11.6 \%$ and $15.5 \%$ respectively. Ten patients died and the cumulative mortality at 1 year, 2 year and 5 year was $0.3 \%, 1.7 \%$ and $3.4 \%$ respectively. Patients of viral and alcoholic mixed etiology and patients with higher baseline MELD score $(\geq 20)$ had a higher rebleeding risk (HR, 6.69 and 5.75, respectively), and patients underwent more sessions of endoscopic treatment had lower variceal recurrence risk (HR 0.81). Baseline presence of portal vein thrombosis and MELD score was highly associated with mortality (HR 8.1 and 8.4 respectively).

Conclusions Recurrent bleeding and mortality have been greatly improved after variceal eradication through EST in cirrhotic patients with EGV. A mixed cirrhotic etiology increased the risk of rebleeding, and portal vein thrombosis and high MELD score increased the risk of mortality.

\section{IDDF2019-ABS-0045 COMPARISON OF SEQUENTIAL, INCREMENTAL AND CONCOMITANT REINTRODUCTION REGIMENS OF ANTI- TUBERCULAR THERAPY(ATT) AFTER INITIAL EPISODE OF ATT HEPATITIS: A SYSTEMATIC REVIEW AND NETWORK META-ANALYSIS}

Praveen Kumar*, Hariom Soni, Shubhra Mishra, Balaji Bellam, Bikash Medhi, Harshal Mandavdhare, Harjeet Singh, Usha Dutta, Vishal Sharma. Postgraduate Institute of Medical Education and Research, India

\subsection{6/gutjnl-2019-IDDFabstracts.247}

Background Various reintroduction regimens are used for reintroducing antitubercular therapy (ATT) in patients who had hepatitis previously related to use of ATT. Reintroduction of ATT in these patients has not been studied systematically.

Methods We performed a systematic review and network meta-analysis of randomized trials to compare the safety of three different reintroduction regimens (Incremental, sequential and concomitant) of ATT using R software with 'gemtc' package. Articles were retrieved from various databases from inception to $28^{\text {th }}$ Dec 2018 with 'chemical and drug-induced liver injury', 'ATT hepatotoxicity', 'drug-induced liver injury' and 'reintroduction of anti-tubercular drugs' as search keywords. We excluded the articles published in languages other than English, case reports, case series and narrative reviews. Recurrence of hepatotoxicity after reintroduction of ATT was taken as the primary outcome. We also compared if the sequence of introduction of isoniazid or rifampin affected the risk of ATT hepatitis. The random effect network model was constructed taking logit likelihood function and heuristically imputed outcome scale assuming uniform standard deviation $(0,2.615)$ as heterogeneity prior. The burn in, inference and thinning were kept at 20000, 100000 and 10 respectively.

Results We found a total of 71 citations in our systematic search, out of which 4 randomized control trials were found eligible and included in the meta-analysis. The rank probability test of network meta-analysis demonstrated that incremental reintroduction therapy has a high chance of number one in all three regimens. Compared with concomitant regimen, incremental regimen was found better (odds ratio [OR] 0.24; 95\% CI 0.017,1.2) then sequential regimen (OR 0.33; 95\% CI $0.033,1.7)$. The point scale reduction factor of both consistency and node-split models was achieved less than 1.05. The deviance information criterion (DIC) and residual deviance of the consistency model were 17.3 and 9.8.

Conclusions The sequential and incremental were better than concomitant. The order of reintroduction of rifampin and isoniazid does not seem to make any difference to the recurrence of ATT hepatitis. 